\title{
Altered Insula Connectivity under MDMA
}

\author{
Ishan C Walpola*,', Timothy Nest', Leor Roseman², David Erritzoe ${ }^{2}$, Amanda Feilding ${ }^{3}$, David J Nutt ${ }^{2}$ and \\ Robin L Carhart-Harris ${ }^{2}$
}

'Department of Psychiatry, McGill University Faculty of Medicine, McGill University, Montreal, Quebec, Canada; ${ }^{2}$ Division of Brain Sciences, Faculty of Medicine, Centre for Neuropsychopharmacology, Imperial College London, London, UK; ${ }^{3}$ The Beckley Foundation, Oxford, UK

\begin{abstract}
Recent work with noninvasive human brain imaging has started to investigate the effects of 3,4-methylenedioxymethamphetamine (MDMA) on large-scale patterns of brain activity. MDMA, a potent monoamine-releaser with particularly pronounced serotonin- releasing properties, has unique subjective effects that include: marked positive mood, pleasant/unusual bodily sensations and pro-social, empathic feelings. However, the neurobiological basis for these effects is not properly understood, and the present analysis sought to address this knowledge gap. To do this, we administered MDMA-HCl (I00 mg p.o.) and, separately, placebo (ascorbic acid) in a randomized, doubleblind, repeated-measures design with twenty-five healthy volunteers undergoing $\mathrm{fMRI}$ scanning. We then employed a measure of global resting-state functional brain connectivity and follow-up seed-to-voxel analysis to the fMRI data we acquired. Results revealed decreased right insula/salience network functional connectivity under MDMA. Furthermore, these decreases in right insula/salience network connectivity correlated with baseline trait anxiety and acute experiences of altered bodily sensations under MDMA. The present findings highlight insular disintegration (ie, compromised salience network membership) as a neurobiological signature of the MDMA experience, and relate this brain effect to trait anxiety and acutely altered bodily sensations - both of which are known to be associated with insular functioning.

Neuropsychopharmacology (2017) 42, 2152-2162; doi:I0.1038/npp.2017.35; published online 29 March 2017
\end{abstract}

\section{INTRODUCTION}

MDMA (3, 4-methylenedioxymethamphetamine) belongs to a unique class of psychoactive compounds-'entactogens'that share similarities in chemical structure with both classical stimulants and psychedelics (Nichols, 1986; Gouzoulis-Mayfrank, 2001). The potent action of MDMA at the serotonin transporter-compared with other stimulants (Bradbury et al, 2014)-is believed to be responsible for much, but not all, of MDMA's unique phenomenology (Liechti and Vollenweider, 2001; van Wel et al, 2011). Common psychological features of the MDMA experience include heightened arousal and perception, pro-sociability, enhanced mood, and feelings of euphoria. Acute physiological effects of MDMA consist of increases in heart rate, blood pressure, body temperature (Vollenweider et al, 1998), and impaired homeostatic control more generally (Dafters and Lynch, 1998) - a phenomenon that has been suggested to involve the insula (Parrott, 2012).

Pharmacological resting-state electroencephalography (EEG; Frei et al, 2001), positron emission tomography (PET; Gamma et al, 2000), and functional magnetic resonance imaging (fMRI; Carhart-Harris et al, 2015;

\footnotetext{
*Correspondence: IC Walpola, Department of Psychiatry, McGill University, 6825 LaSalle Boulevard, Montreal, Quebec, Canada $\mathrm{H} 4 \mathrm{H}$ IR3, Tel: 5 |476620 I0, E-mail: ishan.walpola@mail.mcgill.ca

Received 22 May 2016; revised 5 February 2017; accepted 8 February 2017; accepted article preview online 14 February 2017
}

Roseman et al, 2014) have begun to elucidate the immediate effects of MDMA on the human brain. PET imaging revealed decreased regional cerebral blood flow ( $\mathrm{rCBF}$ ) in areas including the left amygdala, dorsal anterior cingulate, posterior cingulate, medial temporal lobe (MTL), and bilateral insula (Gamma et al, 2000). Gamma et al suggest that these results might represent dysregulation of a functional network that others have since linked to the anxiety phenome (Paulus and Stein, 2006).

In a recent fMRI study using arterial spin labeling (ASL), decreases in cerebral blood flow (CBF) were observed following MDMA administration. These decreases in CBF occurred in MTL structures including the right amygdala and hippocampus, as well as the visual cortex, thalamus and somatosensory cortex (Carhart-Harris et al, 2015). The degree of CBF decreases in the MTL significantly correlated with the subjective intensity of drug effects across subjects. Furthermore, seed-based resting-state functional connectivity (RSFC) using ventromedial prefrontal cortex, amygdala and hippocampal 'seed' regions of interest (ROIs) revealed changes in functional circuitry implicated in anxiety and stress (Carhart-Harris et al, 2015). MDMA-induced RSFC changes with these regions correlated at a trend-level with subjective intensity and positive mood.

Of particular importance to the present study is the prior result of decreased regional brain blood flow in the insula, a region implicated in visceral and somatosensory sensation, following MDMA administration (Gamma et al, 2000). This is intriguing as MDMA is known to produce unusual bodily 
or 'interoceptive' effects, but the neural correlates of these phenomena are not understood. Indeed, altered bodily sensation- potentially underpinned by homeostatic dysregulation-has provided a basis for others to implicate altered functioning of the insula (Parrott, 2012).

For these reasons, we held the prior hypothesis that a datadriven analytic approach may implicate abnormal insula connectivity under MDMA. Using this method, our study would be able to provide the first examination of altered salience network connectivity under MDMA, as the insula is considered an 'integral' hub (Menon and Uddin, 2010) of this network involved in the attribution of environmental/ bodily salience, as well as anxiety and stress (Craig, 2003; Hermans et al, 2011; Seeley et al, 2007). Although other studies examining brain activity under MDMA have not explicitly identified involvement of the salience network, one early PET study identified altered metabolic activity in core hubs of the salience network: the bilateral insula and dorsal anterior cingulate (Gamma et al, 2000). However, no network-based analysis of the functional interconnections between these brain regions under the influence of MDMA has been conducted to date. The two published RSFC studies of MDMA were unable to probe the salience network due to methodological constraints involving either the selection of a priori seed ROIs (Carhart-Harris et al, 2015) that were not specific hubs anchoring the salience network (Bressler and Menon, 2010) or independent components that did not include the salience network (Roseman et al, 2014).

One ambition of the neuroimaging studies described above is to help inform potential therapeutic applications of MDMA, such as its adjunctive use alongside psychotherapy for the treatment of post-traumatic stress disorder (PTSD). The first randomized controlled trials (RCTs) of MDMAassisted psychotherapy for treatment-resistant PTSD demonstrated decreases in both clinician- and participant-rated symptom severity (Mithoefer et al, 2011; Oehen et al, 2013). The efficacy of these interventions persisted at follow-up (Mithoefer et al, 2012), and effect sizes surpassed even those demonstrated by prolonged exposure therapy, the most widely accepted treatment for PTSD (Amoroso and Workman, 2016). Nonetheless, limitations pertaining to modest sample sizes and potential implicit biases warrant cautious interpretation of these preliminary, yet promising results.

In light of growing interest in the use of MDMA as a therapeutic adjunct, the aim of the present study was to use a novel analysis approach to re-explore a previously described resting-state fMRI data set (Carhart-Harris et al, 2015; Roseman et al, 2014). We sought to better understand alterations in the functional neural architecture specific to acute MDMA exposure, by using a novel voxel-to-voxel technique that circumvents some major limitations specific to both seed-based functional connectivity (Carhart-Harris et al, 2015) and independent component analysis (Roseman et al, 2014). The Intrinsic Connectivity Contrast (ICC) has previously been applied to capture novel and unique variations in functional connectivity due to administration of the anesthetic sevoflurane (Martuzzi et al, 2011), providing precedent of its utility in pharmacological resting-state fMRI connectivity analysis.

Unlike traditional seed-based analytic procedures, the ICC does not require geometric definition of a seed ROI (eg, a spherical region of interest of an arbitrary size) at previously reported coordinates. Instead, the ICC allows the seed region for subsequent seed-to-voxel analysis to be defined by the contours of a resultant cluster from a contrast of interest. Unlike traditional methods for independent component analysis of resting-state networks, an arbitrary number of independent components corresponding to spatially independent resting-state networks do not have to be defined for the basis of group- or condition-level contrasts. Instead, functional connectivity of anatomical regions of interest (eg, the insula) displaying network membership with a restingstate network (eg, the salience network) can be examined. Therefore, differences that emerge do not pertain exclusively to network-level attributes, but rather to membership of an anatomical region with functionally defined boundaries and resting-state network membership. Thus, this technique could be of particular importance when investigating functionally heterogeneous, but relatively small regions of the cortex such as the insula, as has previously been demonstrated (Martuzzi et al, 2011). Importantly, however, the ICC is fundamentally an exploratory analytic technique. As such, it is useful for identifying and precisely defining the spatial contours of brain regions with altered functional connectivity to be further interrogated using more traditional seed-based approaches (Martuzzi et al, 2011; Muller et al, 2016a,b).

As previous studies have suggested that MDMA dysregulates a 'neural network' involved in the anxiety phenome, we were further interested in examining the relationship between altered insula connectivity under MDMA and two phenomenological categories: 1) Altered bodily sensation and 2) Trait anxiety. We used altered bodily sensations as a proxy for dysregulated interoception, with interoception understood as 'the sensing of the internal state of one's body' (Tsakiris and Critchley, 2016). Emerging evidence suggests an important link between interoceptive processing and trait anxiety, and that 'interoceptive structure may be a vulnerability factor for anxiety' (Garfinkel et al, 2016). Thus, we would expect dysregulation of an insula network to be associated with greater alterations in bodily sensations (ie, dysregulated interoception) and trait anxiety (ie, anxiety vulnerability). This would provide evidence of a putative neurobiological basis to inform future studies interested in mechanisms underlying the therapeutic use of MDMA as an adjunct in the treatment of psychiatric disorders characterized by anxiety (eg, PTSD).

\section{MATERIALS AND METHODS}

\section{Design}

This study is a novel analysis of a previously published data set (Carhart-Harris et al, 2015; Roseman et al, 2014). As such, detailed descriptions of the design can be found elsewhere (Carhart-Harris et al, 2015). In brief, fMRI data was collected using a within-subjects, double-blind randomized, placebo-controlled design. Each participant was scanned twice, once after placebo and once after MDMA (in counterbalanced order), 7 days apart. A Home Office License was obtained for the storage and handling of a Schedule I drug. A schematic diagram of the scanning procedure is provided below in Figure 1. Only the first 


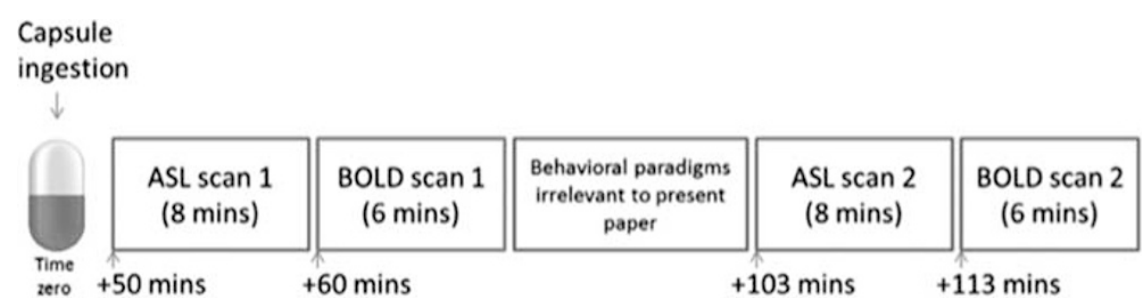

Figure I Schematic showing scanning protocol. Capsules contained Placebo (vitamin C) or 3, 4-methylendediocymethamphetamine (MDMA) hydrochloride ( $100 \mathrm{mg}$ ). Of relevance to the present analysis is the first BOLD scan performed at 60 min following capsule ingestion, outlined in red. BOLD, blood oxygen level dependent. Figure adapted from Carhart-Harris et al, 2015.

resting-state BOLD scan, highlighted in red, was used to avoid any confounding residual or 'carry-over' effects of the intermediate behavioral paradigms on global functional connectivity (Waites et al, 2005).

\section{Participants}

Twenty-five (seven female, mean age $=34 \pm 11$ years) healthy participants with at least one previous experience with MDMA were included in the study sample. None of the participants had consumed MDMA in at least $48 \mathrm{~h}$ (confirmed via urine screen) or recently ingested alcohol (confirmed by a breathalyzer test). Further screening measures and participant demographics are reported elsewhere (Carhart-Harris et al, 2015).

\section{Drug Administration}

MDMA and placebo were administered orally in the form of a capsule. Visits during which MDMA or placebo was consumed were counterbalanced and separated by a 7-day interval. Peak subjective effects were reported $100 \mathrm{~min}$ following MDMA capsule ingestion, consistent with previous observations of plasma t-max (Kolbrich et al, 2008).

\section{Scanning Parameters and Data Acquisition}

Magnetic resonance imaging was performed on a 3-Tesla Siemens Tim Trio (Siemens Healthcare, Erlangen, Germany) using a 32-channel phased array head coil. ADNI-GO MPRAGE parameters with $1 \mathrm{~mm}$ isotropic voxels were used for acquisition of anatomical reference images. BOLDweighted functional images were acquired using a gradient echo planar imaging sequence with $3 \mathrm{~mm}$ isotropic voxels, $\mathrm{TR}=2000 \mathrm{~ms}$, $\mathrm{TE}=31 \mathrm{~ms}$, field-of-view $=192 \mathrm{~mm}, 80^{\circ} \mathrm{flip}$ angle, 36 axial slices in each TR, GRAPPA acceleration $=2$, bandwidth $=2298 \mathrm{~Hz} /$ pixel. Each functional scan for MDMA and placebo lasted $6 \mathrm{~min}$ and was performed 60 -min and 113-min post-capsule ingestion.

\section{Preprocessing}

fMRI preprocessing and analysis were performed using Statistical Parametric Mapping software (SPM12, Wellcome Trust Centre for Neuroimaging, London, UK, http://www.fil. ion.ucl.ac.uk/spm/software/). Preprocessed images were imported into CONN: The Functional Connectivity toolbox (https://www.nitrc.org/projects/conn) in MATLAB for all functional connectivity analyses. A standard preprocessing pipeline was used that included slice-timing correction, rigid body realignment, spatial smoothing with a Gaussian kernel (FWHM) of $6 \mathrm{~mm}$, and all anatomical scans were registered to the Montreal Neurological Institute standard brain space.

In order to best account for motion-related artifacts that can pose a significant confound to observed resting-state correlations (Power et al, 2014), we performed quality control by 'scrubbing' to effectively remove time points with excessive head motion (set at a threshold of $z=3 \mathrm{~mm}$ ). Approaches to scrubbing that actually remove outlier scans impact subsequent resting-state functional connectivity preprocessing steps that assume a continuous time-series (eg, band-pass filtering) by imposing discontinuities. Instead, we opted to implement an approach available in the CONN toolbox, through ART Artifact Detection Tools (https:// www.nitrc.org/projects/artifact_detect/), that effectively 'removes' outlier scans by including them as dummy-coded regressors during the de-noising procedure. Statistical tests were conducted in order to determine whether any group differences could be found in mean motion or scans 'scrubbed' between conditions (see Results section).

Other sources of noise in the BOLD signal from the white matter and cerebrospinal fluid were corrected for by using a principle component-based 'CompCor' method (Murphy et al, 2009; Whitfield-Gabrieli and Nieto-Castanon, 2012). We applied a band-pass filter $(0.008-0.09 \mathrm{~Hz})$ to limit the effect of low-frequency drift and high-frequency noise on the BOLD signal.

\section{Intrinsic Connectivity Contrast}

To identify brain regions showing significantly altered functional connectivity acutely following MDMA compared with placebo administration, we analyzed whole-brain voxelto-voxel connectivity with the intrinsic connectivity contrast (ICC), a network theory approach that has previously been used to elucidate alterations in functional connectivity associated with administration of the anesthetic sevoflurane (Martuzzi et al 2011). The ICC quantifies the presence of, and assigns a weight to, the strength of a connection between any given voxel and every other voxel, computing this metric for every single voxel in the brain (Martuzzi et al, 2011; Scheinost et al, 2012). Individual first-level voxel-to-voxel covariance matrices were computed for each participant for both conditions (MDMA or placebo). This allows for a second-level analysis testing for differences in whole-brain connectivity between conditions without the need to constrain analysis to a priori ROIs based on prior assumptions-allowing functional connectivity to be used as a data- 
driven exploratory tool for the definition of ROIs (Martuzzi et al, 2011). Differences were assessed using a paired t-test with a significance threshold of $p<0.001$ uncorrected, 5voxel cluster threshold (Martuzzi et al, 2011). Others have emphasized that it is critical to keep in mind that the result should not be interpreted in terms of statistical significance, but rather as a threshold for an effect (Muller et al, 2016a,b) to be further interrogated for statistical significance. The resulting clusters provide a global picture of functional connectivity alterations. As such, follow-up seed based functional connectivity utilizing these clusters as ROIs is required to determine the pattern of altered functional connectivity driving this effect.

\section{Follow-up Seed-To-Voxel Functional Connectivity}

In this step, classical seed-based functional connectivity is applied to examine alterations in functional connectivity profiles between conditions using clusters derived from the ICC as a seed ROI. In brief, the average filtered BOLD signal in the ROI is correlated with the BOLD signal in all other voxels in the brain for each condition. The main effect of each condition was calculated to determine intrinsic connectivity network membership of the ICC cluster of interest. Functional connectivity differences between conditions were statistically assessed using a paired t-test. We used a height threshold of $p<0.001$, and a cluster-size threshold of $p<0.05$, corrected using false discovery rate (FDR). In keeping with the previous application of this method (Martuzzi et al, 2011), a significant cluster located in the right insula was used for all subsequent analyses.

\section{ROI-to-ROI Analysis of Primary Salience Network Hubs}

In order to determine whether observed alterations in salience network connectivity were exclusive to the coordinates of the seed region obtained from the ICC or more generally reflective of the salience network, we conducted a
ROI-to-ROI analysis. We defined $6 \mathrm{~mm}$ spherical ROIs at previously reported coordinates (Raichle, 2011) for the dorsal anterior cingulate cortex (dACC) and the bilateral anterior insula (AI)-regions believed to anchor the brain's salience network (Seeley et al, 2007; Menon and Uddin, 2010). The ROI-to-ROI analysis using each seed region was corrected for multiple comparisons using an FDR threshold of $p<0.05$.

\section{Relationship between Changes in Functional Connectivity and Subjective Effects of MDMA}

To explore whether changes in insula RSFC between placebo and MDMA conditions were related to subjective ratings of 'unusual bodily sensations' following the MDMA scan or 'trait anxiety' preceding the scan (ie, at baseline), two correlations were tested, giving a revised statistical threshold of $p<0.025(0.05 / 2)$, corrected with FDR. These specific items were chosen due to prior literature suggesting the critical involvement of insula functioning in processing bodily sensations (Craig, 2003; Critchley et al, 2004) and trait anxiety (Alvarez et al, 2015; Paulus and Stein, 2006).

\section{RESULTS}

\section{Intrinsic Connectivity Contrast}

The ICC (MDMA > placebo) revealed a distributed collection of thirty-six clusters overlapping with the HarvardOxford atlas, exhibiting both increases and decreases in global functional connectivity (Supplement 1). A right insula cluster (Figure 2a; MNI coordinates $+46,+04,+02$ ) was selected from this list as a seed ROI for all subsequent analyses as it allowed us to closely follow a prior application of this method (Martuzzi et al, 2011). Furthermore, alterations in insular functional connectivity following MDMA administration have never been explored despite converging evidence implicating a functional network anchored in the insula in subjective feelings of the body

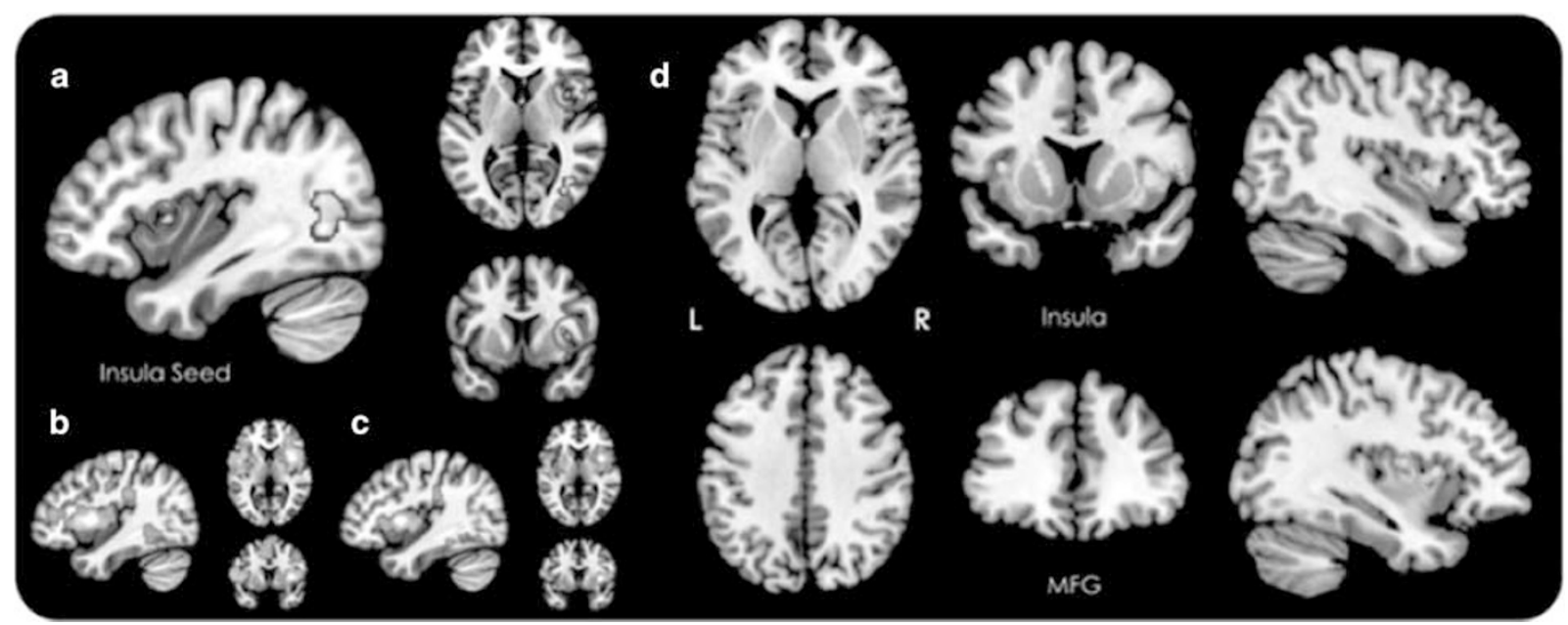

Figure 2 Resting-state functional connectivity (RSFC) after administration of 3, 4-methylenedioxymethamphetamine (MDMA $>$ Placebo). (a) Changes in global RSFC after MDMA ( $p<0.05$, uncorrected; right insula cluster circled in red is used as a seed ROI for all follow-up analyses). (b) Seed-to-voxel RSFC of right insula-anchored salience network in the placebo condition. (c) Seed-to-voxel RSFC of right insula-anchored salience network in the MDMA condition. (d) Changes in right insular seed-to voxel RSFC after MDMA. Increases in RSFC (a) and positive RSFC correlations (b, $c$ ) are shown in warm colors. Decreases in RSFC are shown in cool colors. All seed-to-voxel images (b-d) were cluster corrected at a threshold of $p<0.05$, FDR. FDR, false discovery rate. 
(Craig, 2002, 2003, 2009, 2010, 2011) and anxiety (Alvarez et al, 2015; Grupe and Nitschke, 2013; Paulus and Stein, 2006; Seeley et al, 2007). Therefore, there was a firm rationale for selecting this particular region for subsequent seed-based analyses.

\section{Follow-up Seed-To-Voxel Functional Connectivity}

Condition-specific functional network topography of the insula cluster obtained through ICC for both placebo (Figure 2b) and MDMA (Figure 2c) revealed a functional brain network similar to previous examinations of insular connectivity (Cauda et al, 2011; Deen et al, 2011; Taylor et al, 2009) and overlapping spatially with the so-called 'salience network' (Seeley et al, 2007).

Follow-up seed-to-voxel resting-state functional connectivity comparisons between the MDMA and placebo conditions revealed significantly decreased connectivity (Figure 2d) between the right insula seed and clusters in the right $(+38+12+00)$ and left $(+38+14+08)$ anterior insular cortex, as well as the dorsolateral prefrontal cortex (DLPFC), specifically the left middle frontal gyrus $(+02,+26$, +54 ) under MDMA.

\section{ROI-to-ROI Analysis of Primary Salience Network Hubs}

ROI-to-ROI analysis of the dACC and bilateral AI were defined at independently reported coordinates (Raichle et al, 2011), supporting diminished salience network connectivity in the MDMA condition compared with the placebo condition (Figure 3). When the dACC was seeded, we

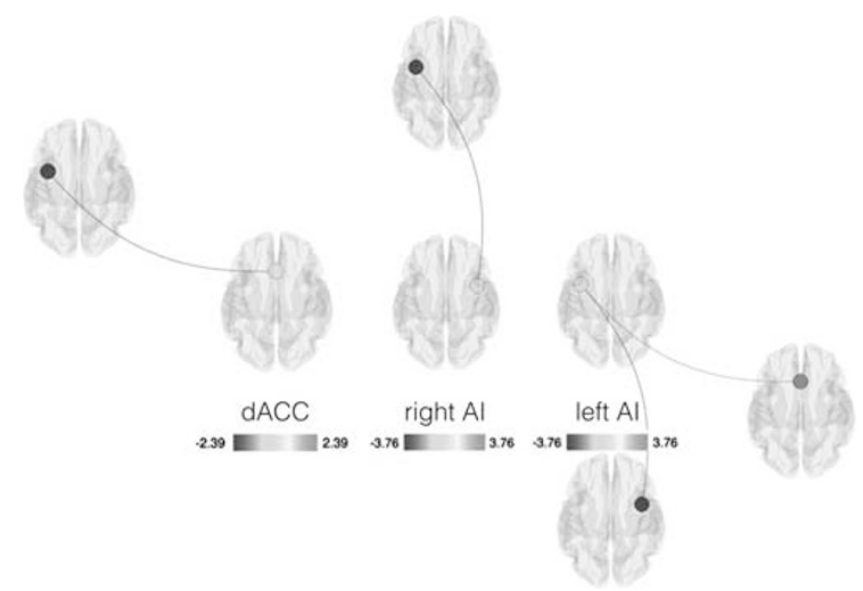

Figure 3 ROI-to-ROI resting-state functional connectivity (RSFC) of salience network hubs after administration of 3, 4-methylenedioxymethamphetamine (MDMA > Placebo). Seed ROls are indicated by bold font. Decreases in RSFC are shown in cool colors. All images were corrected for multiple comparisons at a threshold of $p<0.05$, FDR. Al, anterior insula; $\mathrm{dACC}$, dorsal anterior cingulate cortex; $\mathrm{ROI}$, region of interest. observed significantly decreased connectivity only with the left AI. When the right AI was seeded, we observed significantly decreased connectivity only with the left AI (ie, decreased connectivity with the dACC was not observed, corroborating our seed-to-voxel results within a reduced space of statistical comparisons). Finally, when the left AI was seeded, we observed significantly decreased connectivity with both the right AI and dACC.

\section{Relationship between Changes in Functional Connectivity and Subjective Effects of MDMA}

The group-level results of the relationship between changes in right insula functional connectivity between conditions (MDMA > placebo) are presented for 'trait anxiety' and 'unusual bodily sensations' (Figure 4). Greater trait anxiety was associated with decreased connectivity between the right insula seed region and bilateral DLPFC, specifically the superior frontal gyrus (Figure $4 \mathrm{a} ;+02+24+54$ ) with a Pearson correlation coefficient $\left(R^{2}\right)$ of 0.61 (illustrated in Figure 4c). 'Unusual bodily sensations' were associated with decreased connectivity between the right insula seed region and two clusters: (1) the DLPFC, specifically the right superior frontal gyrus $(+02+20+62)$ and (2) the anterior mid-cingulate gyrus (depicted in Figure $4 \mathrm{~b} ;+08+26+32$ ) with a Pearson correlation coefficient $\left(R^{2}\right)$ of 0.53 (illustrated in Figure 4d). Both correlations survived our revised FDR correction for multiple comparisons. The topographic maps are depicted with a translucent mask of the salience network derived from the right insula seed in the placebo condition to illustrate how the reported clusters of significance overlap and extend beyond the demarcations of this functionally defined network.

\section{Between-Condition Differences in Motion and Scrubbing}

No significant differences were found in the mean motion $(p=0.71)$ or mean scans 'scrubbed' $(p=0.57)$ between the first MDMA and placebo condition.

\section{Between-Subject Differences in Prior MDMA Use}

Due to considerable between-subject variability in history of prior MDMA use, additional linear regression analyses were conducted with the frequency of MDMA use as a nuisance variable. Between-subject differences in prior MDMA use failed to significantly account for any of the betweencondition functional connectivity outcomes reported above.

\section{DISCUSSION}

The present study employed a network theory approach, the Intrinsic Connectivity Contrast (ICC), to elucidate the

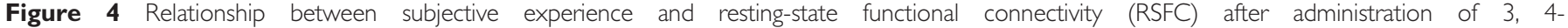

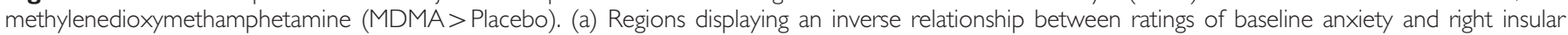

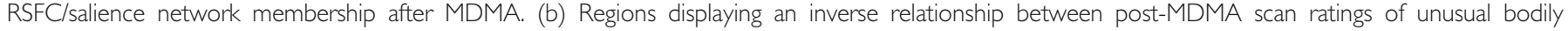

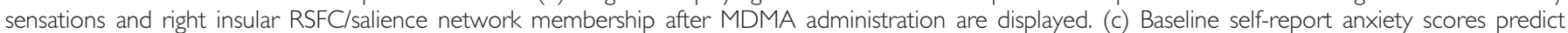

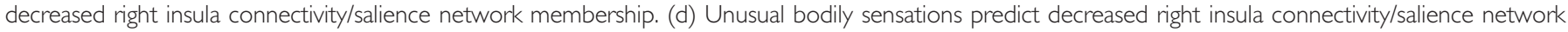
membership. The decreases in RSFC are shown in cool colors. A translucent mask of the salience network (Figure $2 b$ ) is overlaid for display purposes.
} 
impact of MDMA on voxelwise functional brain connectivity. The ICC allows functional connectivity analysis to proceed without the need to first geometrically define a priori ROIs, thus extending the scope of previous efforts
(Carhart-Harris et al, 2015). We observed alterations in functional connectivity between the placebo and MDMA condition in many clusters overlapping with the HarvardOxford Atlas (Supplementary Information S1), notably

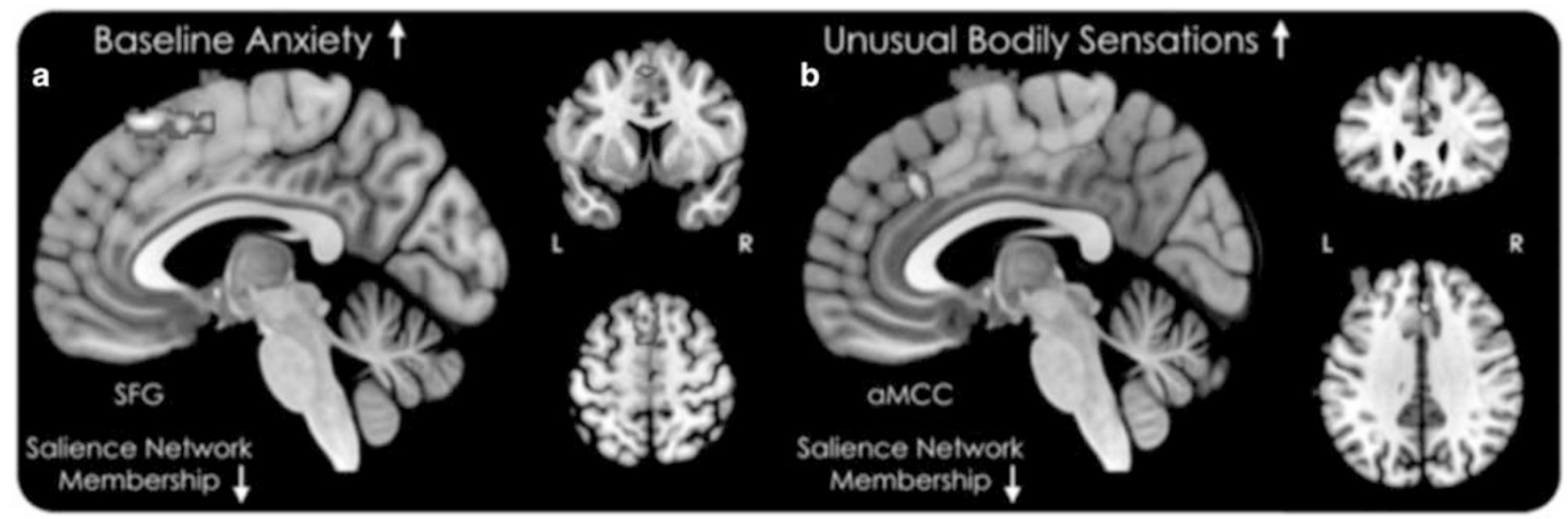

c

Changes in right insula RSFC under MDMA vs. baseline self-report anxiety

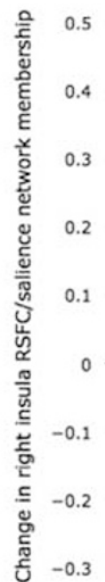

42

44

46

48

50

52

54

Baseline self-report anxiety

d

Changes in right insula RSFC under MDMA vs. unusual bodily sensations

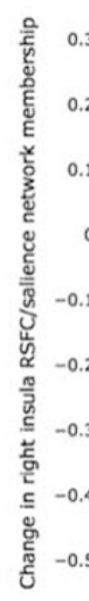

0.3
0.2
0.1
0
-0.1
-0.2
-0.3
-0.4
-0.5
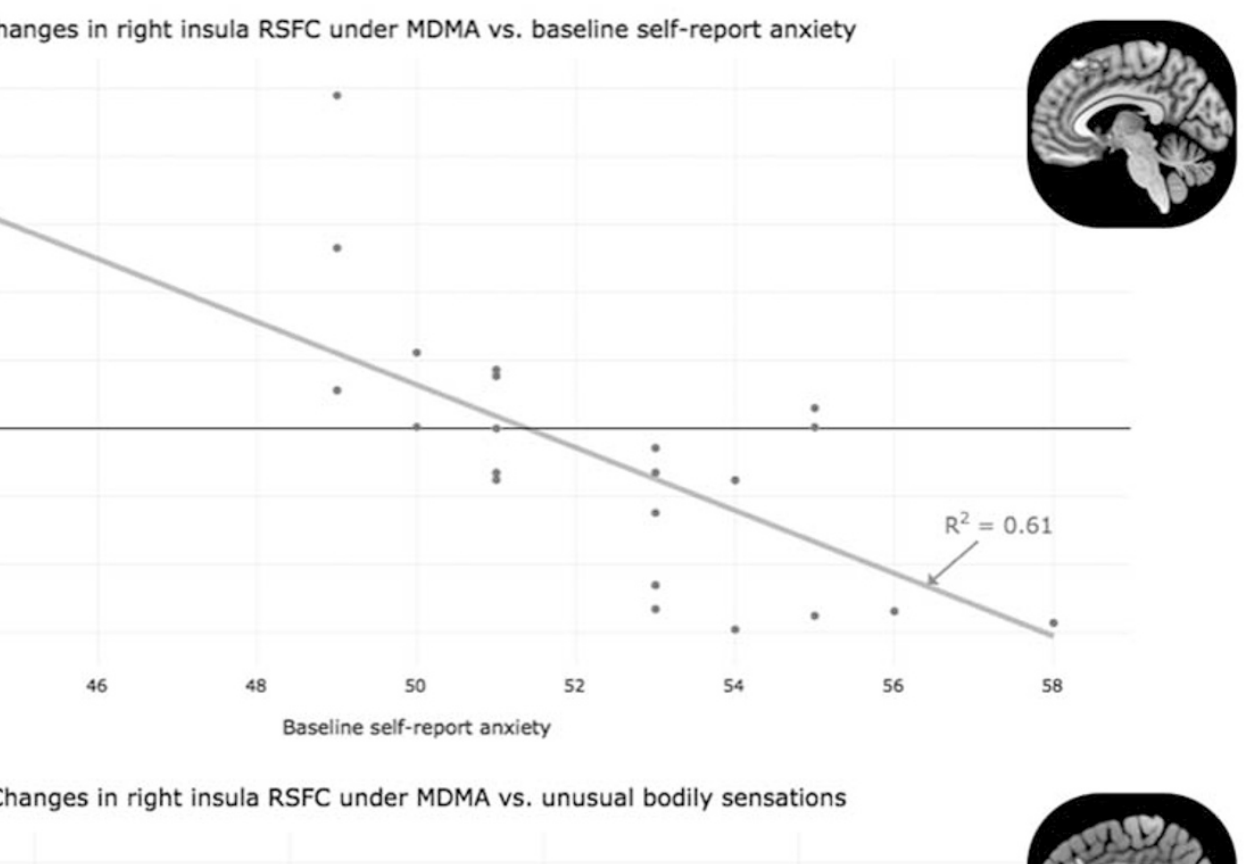

1 
including the right insular cortex (Figure 2a). All further analyses used the insula cluster revealed by the ICC as a seed ROI because it was selected by a prior application of the ICC (Martuzzi et al, 2011), decreased right insular connectivity was strikingly absent from previous fMRI analyses applied to this data set (Carhart-Harris et al, 2015; Roseman et al, 2014), and the insula plays a crucial role in both subjective feelings of the body (Craig, 2002; 2009; 2010; 2011; Critchley et al, 2004) and anxiety (Alvarez et al, 2015; Grupe and Nitschke, 2013; Paulus and Stein, 2006).

When we examined the functional network membership of the right insula cluster using seed-to-voxel functional connectivity in both the MDMA and placebo condition (Figure $2 \mathrm{~b}$ and $\mathrm{c}$ ), we observed a network corresponding to the salience network (Seeley et al, 2007) upon visual inspection. A contrast between conditions (MDMA > placebo) revealed decreased connectivity between the right insula seed ROI, the bilateral anterior insula and portions of the DLPFC (ie, the middle frontal gyrus). We confirmed that decreased salience network connectivity following MDMA administration was not specific to our choice of insula ROI, by conducting an ROI-to-ROI analysis with independent coordinates reported for three hubs of the salience network: the left AI, the right AI and the dACC (Raichle et al, 2011). Of note is the decreased connectivity between the right AI seed and left AI, but not the right AI seed and AACC, even when the search space for differences in statistically significant correlations was reduced from the whole brain to only three salience network ROIs (Figure 3). This suggests that altered right insula connectivity under MDMA might be specific to certain hubs of the salience network, leaving other connections between hubs functionally uncompromised.

Seeley et al, 2007, have previously demonstrated increased connectivity in the salience network (dACC and dorsolateral prefrontal cortex) in association with pre-scan anxiety, interpreted as evidence of trait anxiety coded to some degree in the functional architecture of the salience network (2007). Furthermore, alterations in insular functional connectivity following MDMA administration have never been explored despite converging evidence implicating a functional network anchored in the insula in subjective feelings of the body (Craig, 2002; 2003; 2009; 2010; 2011) and anxiety (Alvarez et al, 2015; Grupe and Nitschke, 2013; Paulus and Stein, 2006; Seeley et al, 2007). As such, we endeavored to investigate how differences in right insula functional connectivity under MDMA (compared with placebo) might be related to individual differences in trait anxiety. Interestingly, we found that greater decreases in right insular connectivity within a dorsolateral prefrontal region-the superior frontal gyrus-in individuals who had greater trait anxiety. The cluster of deactivation that negatively correlated with levels of trait anxiety overlapped and extended anteriorly beyond the boundaries of the salience network we functionally defined.

Although speculative, it is intriguing to consider whether the observed correlation between baseline trait anxiety and MDMA-induced decreases in salience network connectivityspecifically between the right insula and superior frontal gyrus-might reflect the action of MDMA on a putative functional network coding trait anxiety (Seeley et al, 2007). This would suggest a candidate mechanism (ie, pharmacologically attenuated salience network connectivity) for the therapeutic role of MDMA in clinical syndromes with anxiety as a core component (eg, PTSD). In line with such a possibility, meta-analyses of the functional neuroimaging of anxiety have found heightened insula activation in PTSD, Social Anxiety Disorder and Specific Phobia compared with controls (Etkin and Wager, 2007), and in PTSD compared with both trauma-naïve and traumaexposed controls (Patel et al, 2012). Increased salience network connectivity has also been documented in a host of anxiety disorders, notably including PTSD (Peterson et al, 2014). Furthermore, propranolol-another pharmacological agent investigated as an adjunct for PTSD therapy (Brunet et al, 2008, but see Wood et al, 2015; Steenen et al, 2016)-decreases connectivity within the salience network (Hermans et al, 2011). Thus, it is worth considering whether action at the salience network might be an important component of the mechanism by which MDMA operates in MDMA-assisted psychotherapy for PTSD (Mithoefer et al, 2011; Mithoefer et al, 2012; Oehen et al, 2013).

It should be noted that MDMA is not considered generally anxiolytic, as it has been shown to increase self-reported state anxiety (Baggott et al, 2016; Bedi and de Wit, 2011). Thus, the acute therapeutic effect of MDMA may specifically target neural substrates coding trait, rather than state, anxiety (ie, the salience network). Future studies should specifically investigate this possibility, examining variations in the relationship between altered neural connectivity under MDMA and state $v s$ trait anxiety, both acutely and in the long term.

Based on the unique phenomenology of MDMA, we hypothesized that unusual bodily sensations might be related to dysregulated interoceptive processing (Critchley et al, 2004; Seth, 2013; De Wit et al, 2015) served by a body awareness network anchored in the right anterior insula (Craig, 2005). Thus, we investigated the correlation between self-reports of unusual bodily sensations and MDMAinduced decreases in right insula-anchored salience network connectivity. Our results revealed decreased right insular connectivity with the right SFG and anterior mid-cingulate cortex (aMCC). While decreased connectivity with the right SFG was also associated with greater levels of trait anxiety, decreased connectivity with the aMCC, a region implicated in body awareness (Medford and Critchley, 2010; Vandenbergh et al, 2005), was uniquely related to awareness of unusual bodily sensations. As interoceptive awareness has been found to mediate the relationship between trait anxiety and the intensity of unpleasant feelings (Pollatos et al, 2007), our results raise the possibility that MDMA might attenuate unpleasant feelings-and conversely produce its characteristic positive feelings (Liechti and Vollenweider, 2000)-by decreasing the integrity of a neural network important for encoding trait anxiety and supporting interoceptive awareness.

It has previously been demonstrated that inductions of sad mood increase functional connectivity between the right insula and cingulate cortex (Harrison et al, 2008), and our result supports the possibility that MDMA disrupts the functional circuitry supporting certain negative affective states by compromising the interoceptive network that supports awareness of such feelings (Craig, 2009). Furthermore, there is a developing theoretical basis (Garfinkel et al, 2016; Seth, 2013) to suggest that compromised functional 
integrity of this network might simultaneously give rise to subjective beliefs of unusual bodily sensations typical of the MDMA experience.

Converging evidence of the salience network's importance in schizophrenia (Menon, 2011; Palaniyappan et al, 2013) and altered salience network connectivity following dopaminergic challenge (ie, connectivity is increased by L-dopa and decreased by haloperidol; Cole et al, 2013) may initially seem at odds with our demonstration that MDMA alters insula/salience network connectivity. Human research suggests that serotonin mediates the majority of physiological and psychosocial effects of MDMA (Liechti et al, 2000a, b), while the effect of dopaminergic (D2) blockade was primarily limited to attenuating euphoria (Liechti and Vollenweider, 2000). However, a complex interaction between neuromodulators is likely to mediate salience network functioning. Indeed, recent work has suggested that epigenetic modification (ie, increased methylation) of the serotonin transporter gene (SLC6A4) promoter region predicts heightened insula reactivity (Frodle et al, 2015) and salience network connectivity (Muehlhan et al, 2015). This phenomenon also has relevance for clinical syndromes, such as PTSD, as SLC6A4 methylation has been linked to unresolved trauma (van Ijzendoorn et al, 2010). Thus, an emerging relationship between epigenetic modulation of the serotonin transporter gene, stress/anxiety-related clinical pathology, and the salience network suggests that we should look beyond the confines of the dopaminergic system when thinking about salience network functioning. Future neuroimaging studies incorporating an acute MDMA challenge plus pretreatment with selective dopaminergic (eg, haloperidol, D2) and/or serotonergic (eg, ketanserin, 5-HT2A) receptor antagonists would help to elucidate the respective contributions of these neuromodulatory systems on the behavior of specific brain networks in both healthy individuals and those with anxiety/ trauma-related disorders (eg, PTSD).

Previous resting-state studies examining functional brain networks under MDMA have not implicated the insula (Carhart-Harris et al, 2015; Roseman et al, 2014), and studies that have demonstrated insula involvement have not directly examined functional networks (Gamma et al, 2000). One reason for this could be that ventromedial prefrontal cortex, hippocampus and amygdala functional connectivity with the insula is not altered under MDMA (Carhart-Harris et al, 2015), if functional coupling between these regions is even expected in the first place. Indeed, of these anatomical regions, only the dorsal portion of the amygdala is thought to belong to the salience network (Seeley et al, 2007; Bickart et al, 2014). Furthermore, we did not find altered insula connectivity with any of these regions under MDMA in the present study. Roseman et al, selected 10 independent components of interest corresponding to functional network labels from the BrainMap database (6 components from the analysis were determined to be non-neural noise), but none were identified as corresponding to the salience network (2014) and only inter-network connectivity was examined. The only significant inter-network alteration under MDMA did not involve the insula. Finally, Gamma et al used positron emission tomography, and although they found a constellation of activations and deactivations under MDMA that they infer reflects a functional network, no formal network analysis approach was applied (2000). Therefore, none of these approaches were able to identify altered insulaanchored functional network connectivity under MDMA due to their respective methodological constraints.

Thus, our study represents the first synthesis of these lines of research, demonstrating decreased insula/salience network functional connectivity under MDMA and linking this to baseline levels of trait anxiety and changes in interoception. In light of recent hypotheses suggesting an insular view of anxiety' (Paulus and Stein, 2006), further understanding of how MDMA affects the insula might be crucial to elucidating the neurobiological underpinnings of re-emerging interest in MDMA as a therapeutic adjunct to psychotherapy in the treatment of anxiety disorders including PTSD (Mithoefer et al, 2011; Oehen et al, 2013, see Amoroso and Workman, 2016 for a preliminary meta-analysis).

\section{Limitations and Future Directions}

Although the ICC allows for a data-driven characterization of global alterations in functional connectivity, the subsequent selection of one of these clusters of significance (on relatively subjective criteria) for follow-up seed-to-voxel functional connectivity analysis is one notable limitation of the method. Nonetheless, it does represent a principled approach for empirically constraining seed ROI selection for exploratory analyses in novel neuroimaging contexts. Furthermore, in our study we selected an insular cluster for follow-up seed-to-voxel functional connectivity analysis to closely follow a previous implementation of the procedure (Martuzzi et al, 2011), ensuring our selection criteria was not arbitrary. As outlined, this procedure represents a circular analysis necessary to determine the specific patterns of altered functional connectivity (determined using seed-tovoxel functional connectivity) that drive the ICC. However, we did confirm that MDMA alters insula connectivity with core regions of the salience network even when the central coordinates and spatial contours of insula regions of interest differed from those obtained from the ICC, supporting the likelihood that altered insula connectivity under MDMA is not exclusive to the spatial boundaries of the region of interest determined by the ICC.

Our study also relied on self-report ratings of anxiety and bodily experience, which may be susceptible to bias based on expectations about the effects of MDMA. Future research should employ validated physiological measures (Critchley et al, 2003) and behavioral paradigms (Garfinkel et al, 2016) that probe anxiety and interoception to provide additional objective support to subjective assessments of these phenomena. Another means to circumvent the limitation of selfreport questionnaires in future studies would be to employ automated speech-based analytic approaches (eg, Latent Semantic Analysis) during periods of free-speech after MDMA (Bedi et al, 2014) and link semantic proximity to concepts of interest such as 'anxiety' or 'body' with functional patterns of brain activity-a strategy in line with the research program of neurophenomenology (Varela et al, 1996; Lutz and Thompson, 2003). Such measures could provide a more fine-grained and precise estimate of interoceptive sensation than the self-report measures employed herein, which may have overlapped with sensory domains such as haptics or proprioception.

(1) 
It is important to note that the strength of our reported Pearson correlation coefficients should be interpreted with caution, as they are computed using average functional connectivity in clusters derived from the association between decreases in right insular functional connectivity and the respective subjective metrics (ie, self-report baseline anxiety and unusual bodily sensations). Thus, non-independence between the cluster-forming analyses and calculation of correlation coefficients necessitate future studies with larger cohorts to employ cross-validation between independent samples. Only then can the generalizability of the associations observed here be confidently established.

As we did not mechanically record measurements of alterations in physiological markers such as heart rate or blood pressure, we cannot rule out the possibility that these parameters that have been observed to increase under MDMA (Vollenweider et al, 1998), rather than awareness of them (i.e, interoception), drove alterations in insula functional connectivity. While activity of the insula has been linked to exercise-induced hypertension (Williamson et al, 1999), bradycardia (Volkow et al, 2000), and heart-rate variability (Critchley et al, 2003; Chang et al, 2013), we are not aware of any studies that have found low-level changes in physiology (eg, tachycardia or hypertension) to change insula connectivity per se (ie, when taking the insular cortex as a seed region of interest).

Our study employed a measure of interoceptive sensibility (ie, subjective belief/awareness) based on self-report measures, but other dimensions such as interoceptive accuracy (ie, objective performance on a behavioral task) or interoceptive metacognition (ie, awareness of performance aptitude) are also important facets of interoception (Garfinkel et al, 2016). Future neuroimaging studies investigating MDMA should collect these measures during scanning in order to determine their respective contributions to altered brain function under MDMA.

The ineffectiveness of blinding needs to be highlighted as a limitation of our study, and is described at length elsewhere (Carhart-Harris et al, 2015). Notably, participants correctly determined when they had received MDMA or placebo in $90 \%(45 / 50)$ of study days and the research team predicted correctly in $96 \%(48 / 50)$ of the study days (Carhart-Harris et al, 2015). Ineffective blinding is a common problem that is difficult to surmount in neuroimaging studies of substances with potent acute subjective effects. Strategies to improve the effectiveness of blinding, such as including an active placebo condition or recruiting only drug-naïve participants are not always easy to implement-and to enable simple betweencondition comparisons, a drug-free control condition is often favorable.

In conclusion, the present study demonstrated decreased salience network functional connectivity following MDMA administration that was linked to the characteristic phenomenology of MDMA, namely, the experience of unusual bodily sensations, as well as trait anxiety - which may be especially sensitive to modulation via MDMA (Mithoefer et al, 2011). Nevertheless, much work remains to be done in order to further elucidate the nuances of this neurobiological effect and extend these findings to clinical contexts. With recent observations that the salience network comprises a 'common core' of brain areas affected by major psychiatric illnesses (Downar et al, 2015), pharmacological agents that have a notable action on this functional brain network may be useful for the future study of the etiology, pathophysiology and potential treatment of major psychiatric disorders.

\section{FUNDING AND DISCLOSURE}

The authors declare no conflict of interest.

\section{ACKNOWLEDGMENTS}

We thank Tara Campbell and three anonymous reviewers for helpful comments on earlier versions of this paper. This research was supported by funding from the British public service broadcast station Channel 4 and was performed as part of a Beckley Foundation-Imperial College research program. Screenings for this research were performed at the NIHR/Wellcome Trust Imperial Clinical Research Facility.

\section{REFERENCES}

Alvarez RP, Kirlic N, Misaki M, Bodurka J, Rhudy JL, Paulus MP et al (2015). Increased anterior insula activity in anxious individuals is linked to diminished perceived control. Transl Psychiatry 5: e591.

Amoroso T, Workman M (2016). Treating posttraumatic stress disorder with MDMA-assisted psychotherapy: a preliminary meta-analysis and comparison to prolonged exposure therapy. J Psychopharmacol 30: 595-600.

Baggott MJ, Coyle JR, Siegrist JD, Garrison KJ, Galloway GP, Mendelson JE (2016). Effects of 3, 4-methylenedioxymethamphetamine on socioemotional feelings, authenticity, and autobiographical disclosure in healthy volunteers in a controlled setting. J Psychopharmacol (Oxford) 30: 378-387.

Bedi G, de Wit H (2011). Individual differences in acute responses to MDMA in humans: effects of sex and past ecstasy use. Open Addict J 4: 6-7.

Bedi G, Cecchi GA, Slezak DF, Carrillo F, Sigman M, de Wit H (2014). A window into the intoxicated mind? Speech as an index of psychoactive drug effects. Neuropsychopharmacology 39: 2340-2348.

Bickart KC, Dickerson BC, Barrett LF (2014). The amygdala as a hub in brain networks that support social life. Neuropsychologia 63: $235-248$

Bradbury S, Bird J, Colussi-Mas J, Mueller M, Ricaurte G, Schenk S (2014). Acquisition of MDMA self-administration: pharmacokinetic factors and MDMA-induced serotonin release. Addict Biol 19: $874-884$.

Bressler SL, Menon V (2010). Large-scale brain networks in cognition: emerging methods and principles. Trends Cogn Sci 14: 277-290.

Brunet A, Orr SP, Tremblay J, Robertson K, Nader K, Pitman RK (2008). Effect of post-retrieval propranolol on psychophysiologic responding during subsequent script-driven traumatic imagery in post-traumatic stress disorder. J Psychiatric Res 42: 503-506.

Carhart-Harris RL, Murphy K, Leech R, Erritzoe D, Wall MB, Ferguson B et al (2015). The effects of acutely administered 3, 4methylenedioxymethamphetamine on spontaneous brain function in healthy volunteers measured with arterial spin labeling and blood oxygen level-dependent resting state functional connectivity. Biol Psychiatry 78: 554-562.

Cauda F, D'Agata F, Sacco K, Duca S, Geminiani G, Vercelli A (2011). Functional connectivity of the insula in the resting brain. Neuroimage 55: 8-23. 
Chang C, Metzger CD, Glover GH, Duyn JH, Heinze HJ, Walter M (2013). Association between heart rate variability and fluctuations in resting-state functional connectivity. Neuroimage 68: 93-104.

Cole DM, Oei NY, Soeter RP, Both S, van Gerven JM, Rombouts SA et al (2013). Dopamine-dependent architecture of corticosubcortical network connectivity. Cereb Cortex 23: 1509-1516.

Craig AD (2002). How do you feel? Interoception: the sense of the physiological condition of the body. Nat Rev Neurosci 3: 655-666.

Craig AD (2003). Interoception: the sense of the physiological condition of the body. Curr Opin Neurobiol 13: 500-505.

Craig AD (2005). Forebrain emotional asymmetry: a neuroanatomical basis? Trends Cogn Sci 9: 566-571.

Craig AD (2009). How do you feel-now? the anterior insula and human awareness. Nat Rev Neurosci 10: 59-70.

Craig AD (2010). The sentient self. Brain Struc Func 214: 563-577.

Craig AD (2011). Significance of the insula for the evolution of human awareness of feelings from the body. Ann NY Acad Sci 1225: 72-82.

Critchley HD, Mathias CJ, Josephs O, O'doherty J, Zanini S, Dewar BK et al (2003). Human cingulate cortex and autonomic control: converging neuroimaging and clinical evidence. Brain 126: 2139-2152.

Critchley HD, Wiens S, Rotshtein P, Öhman A, Dolan RJ (2004). Neural systems supporting interoceptive awareness. Nat Neurosci 7: 189-195.

De Wit H, Gorka SM, Phan KL (2015). The Ups and Downs of 3, 4Methylenedioxymethamphetamine: linking subjective effects to spontaneous brain function. Biol Psychiatry 78: 519-521.

Deen B, Pitskel NB, Pelphrey KA (2011). Three systems of insular functional connectivity identified with cluster analysis. Cereb Cortex 21: 1498-1506.

Downar J, Blumberger DM, Daskalakis ZJ (2015). The neural crossroads of psychiatric illness: an emerging target for brain stimulation. Trends Cogn Sci 20: 107-120.

Dafters RI, Lynch E (1998). Persistent loss of thermoregulation in the rat induced by 3, 4-methylenedioxymethamphetamine (MDMA or 'Ecstasy') but not by fenfluramine. Psychopharmacology 138: 207-212.

Etkin A, Wager TD (2007). Functional neuroimaging of anxiety: a meta-analysis of emotional processing in PTSD, social anxiety disorder, and specific phobia. Am J Psychiatry 164: 1476-1488.

Frei E, Gamma A, Pascual-Marqui R, Lehmann D, Hell D, Vollenweider FX (2001). Localization of MDMA-induced brain activity in healthy volunteers using low resolution brain electromagnetic tomography (LORETA). Hum Brain Mapp 14: $152-165$

Frodl T, Carballedo A, Vaisheva F, Meaney J (2015). DNA methylation of the serotonin transporter gene (SLC6A4) is associated with brain function involved in processing emotional stimuli. J Psychiatry Neurosci 40: 296.

Gamma A, Buck A, Berthold T, Hell D, Vollenweider FX (2000). 3, 4-Methylenedioxymethamphetamine (MDMA) modulates cortical and limbic brain activity as measured by [H215O]-PET in healthy humans. Neuropsychopharmacology 23: 388-395.

Garfinkel SN, Seth AK, Barrett AB, Suzuki K, Critchley HD (2015). Knowing your own heart: distinguishing interoceptive accuracy from interoceptive awareness. Biol Psychol 104: 65-74.

Garfinkel SN, Tiley C, O'Keeffe S, Harrison NA, Seth AK, Critchley HD (2016). Discrepancies between dimensions of interoception in autism: implications for emotion and anxiety. Biol Psychol 114: 117-126.

Gouzoulis-Mayfrank E (2001). Differential actions of an entactogen compared to a stimulant and a hallucinogen in healthy humans. Heffter Rev Psychedelic Res 2: 64-72.

Grupe DW, Nitschke JB (2013). Uncertainty and anticipation in anxiety: an integrated neurobiological and psychological perspective. Nat Rev Neurosci 14: 488-501.
Harrison BJ, Pujol J, Ortiz H, Fornito A, Pantelis C, Yücel M (2008). Modulation of brain resting-state networks by sad mood induction. PLoS One, 3: e1794.

Hermans EJ, van Marle HJ, Ossewaarde L, Henckens MJ, Qin S, van Kesteren MT et al (2011). Stress-related noradrenergic activity prompts large-scale neural network reconfiguration. Science 334: 1151-1153.

Hysek CM, Schmid Y, Simmler LD, Domes G, Heinrichs M, Eisenegger $C$ et al (2013). MDMA enhances emotional empathy and prosocial behavior. Soc Cogn Affect Neurosci 9: 1645-1652.

Kolbrich EA, Goodwin RS, Gorelick DA, Hayes RJ, Stein EA, Huestis MA (2008). Plasma pharmacokinetics of 3, 4-methylenedioxymethamphetamine after controlled oral administration to young adults. Ther Drug Monitor 30: 320.

Liechti ME, Baumann C, Gamma A, Vollenweider FX (2000a). Acute psychological effects of 3, 4-methylenedioxymethamphetamine (MDMA,'Ecstasy') are attenuated by the serotonin uptake inhibitor citalopram. Neuropsychopharmacology 22: 513-521.

Liechti ME, Saur MR, Gamma A, Hell D, Vollenweider FX (2000b). Psychological and physiological effects of MDMA ('Ecstasy') after pretreatment with the 5-HT2 antagonist ketanserin in healthy humans. Neuropsychopharmacology 23: 396-404.

Liechti ME, Vollenweider FX (2000). Acute psychological and physiological effects of MDMA ('Ecstasy') after haloperidol pretreatment in healthy humans. Eur Neuropsychopharmacol 10: 289-295.

Liechti ME, Vollenweider FX (2001). Which neuroreceptors mediate the subjective effects of MDMA in humans? A summary of mechanistic studies. Human Psychopharmacology: Clinical and Experimenta 16: 589-598.

Lutz A, Thompson E (2003). Neurophenomenology integrating subjective experience and brain dynamics in the neuroscience of consciousness. I Conscious Stud 10: 31-52.

Martuzzi R, Ramani R, Qiu M, Shen X, Papademetris X, Constable RT (2011). A whole-brain voxel based measure of intrinsic connectivity contrast reveals local changes in tissue connectivity with anesthetic without a priori assumptions on thresholds or regions of interest. Neuroimage 58: 1044-1050.

Medford N, Critchley HD (2010). Conjoint activity of anterior insular and anterior cingulate cortex: awareness and response. Brain Struct Funct 214: 535-549.

Menon V (2011). Large-scale brain networks and psychopathology: a unifying triple network model. Trends Cogn Sci 15: 483-506.

Menon V, Uddin LQ (2010). Saliency, switching, attention and control: a network model of insula function. Brain Struct Func 214: 655-667.

Mithoefer MC, Wagner MT, Mithoefer AT, Jerome L, Doblin R (2011). The safety and efficacy of \pm 3 , 4-methylenedioxymethamphetamine-assisted psychotherapy in subjects with chronic, treatment-resistant posttraumatic stress disorder: the first randomized controlled pilot study. J Psychopharmacol 25: 439-452.

Mithoefer MC, Wagner MT, Mithoefer AT, Jerome L, Martin SF, Yazar-Klosinski B et al (2012). Durability of improvement in posttraumatic stress disorder symptoms and absence of harmful effects or drug dependency after 3, 4-methylenedioxymethamphetamine-assisted psychotherapy: a prospective long-term follow-up study. J Psychopharmacol 27: 28-39.

Muehlhan M, Kirschbaum C, Wittchen HU, Alexander N (2015). Epigenetic variation in the serotonin transporter gene predicts resting state functional connectivity strength within the saliencenetwork. Hum Brain Mapp 36: 4361-4371.

Muller AM, Mérillat S, Jäncke L (2016a). Older but still fluent? Insights from the intrinsically active baseline configuration of the aging brain using a data driven graph-theoretical approach. NeuroImage 127: 346-362.

Muller AM, Mérillat S, Jäncke L (2016b). Small changes, but huge impact? The right anterior insula's loss of connection strength 
during the transition of old to very old age. Front Aging Neurosci 8: 86 .

Murphy K, Birn RM, Handwerker DA, Jones TB, Bandettini PA (2009). The impact of global signal regression on resting state correlations: are anti-correlated networks introduced? Neuroimage 44: 893-905.

Nichols DE (1986). Differences between the mechanism of action of MDMA, MBDB, and the classic hallucinogens. Identification of a new therapeutic class: entactogens. J Psychoactive Drugs 18: 305-313.

Oehen P, Traber R, Widmer V, Schnyder U (2013). A randomized, controlled pilot study of MDMA ( \pm 3 , 4-methylenedioxymethamphetamine)-assisted psychotherapy for treatment of resistant, chronic post-traumatic stress disorder (PTSD). J Psychopharmacol 27: 40-52.

Palaniyappan L, Simmonite M, White TP, Liddle EB, Liddle PF (2013). Neural primacy of the salience processing system in schizophrenia. Neuron 79: 814-828.

Patel R, Spreng RN, Shin LM, Girard TA (2012). Neurocircuitry models of posttraumatic stress disorder and beyond: a metaanalysis of functional neuroimaging studies. Neurosci Biobehav Rev 36: $2130-2142$.

Paulus MP, Stein MB (2006). An insular view of anxiety. Biol Psychiatry 60: 383-387.

Parrott AC (2012). MDMA and temperature: a review of the thermal effects of 'Ecstasy' in humans. Drug Alcohol Depend 121: 1-9.

Peterson A, Thome J, Lanius RA (2014). Resting-state neuroimaging studies: a new way of identifying differences and similarities among the anxiety disorders? Can J Psychiatry 59: 294.

Pollatos O, Traut-Mattausch E, Schroeder H, Schandry R (2007). Interoceptive awareness mediates the relationship between anxiety and the intensity of unpleasant feelings. J Anxiety Disord 21: 931-943.

Power JD, Mitra A, Laumann TO, Snyder AZ, Schlaggar BL, Petersen SE (2014). Methods to detect, characterize, and remove motion artifact in resting state fMRI. Neuroimage 84: 320-341.

Raichle ME (2011). The restless brain. Brain Connect 1: 3-12.

Roseman L, Leech R, Feilding A, Nutt DJ, Carhart-Harris RL (2014). The effects of psilocybin and MDMA on between-network resting state functional connectivity in healthy volunteers. Front Hum Neurosci 8: 204-204.

Scheinost D, Benjamin J, Lacadie CM, Vohr B, Schneider KC, Ment LR et al (2012). The intrinsic connectivity distribution: a novel contrast measure reflecting voxel level functional connectivity. Neuroimage 62: 1510-1519.

Seeley WW, Menon V, Schatzberg AF, Keller J, Glover GH, Kenna $\mathrm{H}$ et al (2007). Dissociable intrinsic connectivity networks for salience processing and executive control. J Neurosci 27: 2349-2356.

Seth AK (2013). Interoceptive inference, emotion, and the embodied self. Trends Cogn Sci 17: 565-573.

Steenen SA, van Wijk AJ, van der Heijden GJ, van Westrhenen R, de Lange J, de Jongh A (2016). Propranolol for the treatment of anxiety disorders: systematic review and meta-analysis. J Psychopharmacol 30: 128-139.

Taylor KS, Seminowicz DA, Davis KD (2009). Two systems of resting state connectivity between the insula and cingulate cortex. Hum Brain Mapp 30: 2731-2745.

Tsakiris M, Critchley H (2016). Interoception beyond homeostasis: affect, cognition and mental health. Philos Trans $R$ Soc Lond $B$ Biol Sci 371: 20160002.

Vandenbergh J, DuPont P, Fischler B, Bormans G, Persoons P, Janssens J et al (2005). Regional brain activation during proximal stomach distention in humans: a positron emission tomography study. Gastroenterology 128: 564-573.

Varela FJ (1996). Neurophenomenology: a methodological remedy for the hard problem. J Conscious Stud 3: 330-349.

van IJzendoorn $\mathrm{MH}$, Caspers $\mathrm{K}$, Bakermans-Kranenburg $\mathrm{MJ}$, Beach SR, Philibert R (2010). Methylation matters: interaction between methylation density and serotonin transporter genotype predicts unresolved loss or trauma. Biol Psychiatry 68: 405-407.

van Wel JHP, Kuypers KPC, Theunissen EL, Bosker WM, Bakker K, Ramaekers JG (2011). Blockade of 5-HT2 receptor selectively prevents MDMA-induced verbal memory impairment. Neuropsychopharmacology 36: 1932-1939.

Vollenweider FX, Gamma A, Liechti M, Huber T (1998). Psychological and cardiovascular effects and short-term sequelae of MDMA ('ecstasy') in MDMA-naive healthy volunteers. Neuropsychopharmacology 19: 241-251.

Volkow ND, Wang GJ, Fowler JS, Logan J, Gatley JS, Pappas NR et al (2000). Increased activity of the temporal insula in subjects with bradycardia. Life Sci 67: 2213-2220.

Waites AB, Stanislavsky A, Abbott DF, Jackson GD (2005). Effect of prior cognitive state on resting state networks measured with functional connectivity. Hum Brain Mapp 24: 59-68.

Williamson JW, McColl R, Mathews D, Ginsburg M, Mitchell JH (1999). Activation of the insular cortex is affected by the intensity of exercise. J Appl Physiol 87: 1213-1219.

Wood NE, Rosasco ML, Suris AM, Spring JD, Marin MF, Lasko NB et al (2015). Pharmacological blockade of memory reconsolidation in posttraumatic stress disorder: three negative psychophysiological studies. Psychiatry Res 225: 31-39.

Whitfield-Gabrieli S, Nieto-Castanon A (2012). Conn: a functional connectivity toolbox for correlated and anticorrelated brain networks. Brain Connect 2: 125-141.

Supplementary Information accompanies the paper on the Neuropsychopharmacology website (http://www.nature.com/npp) 